\title{
Numerical Solution of Singularly Perturbed BVPs using an Optimal Fitted One-Step Integration Scheme via Initial Value Method
}

\author{
E. R. El-Zahar ${ }^{1,2}$ \\ ${ }^{1}$ Department of Mathematics, College of Science and Humanities in Al-Kharj,Prince Sattam bin Abdulaziz University, \\ P.O. Box 83, Al-Kharj, 11942, Saudi Arabia. \\ ${ }^{2}$ Department of Basic Engineering Science, Faculty of Engineering, Shebin El-Kom, 32511, Menofia University, Egypt. \\ *Corresponding Author: ORCID: 0000-0001-7266-1893(E. R. El-Zahar)
}

\begin{abstract}
In this paper, a numerical solution of singularly perturbed BVPs (SPBVPs) using an optimal fitted one-step integration scheme via initial value method is present. The original second order SPBVP is replaced by an asymptotically approximate first order initial value singularly perturbed problem (IVSPP) and solved using an optimal fitted one-step integration scheme. The error analysis is present. Some SPBVPs are solved and the numerical results confirm that the suggested method is accurate and efficient in solving the considered problems.
\end{abstract}

Keywords: Singularly perturbed BVPs, Initial value methods, Exponentially fitted finite difference schemes.

\section{INTRODUCTION}

Singularly perturbed boundary value problems (SPBVPs) are common in applied sciences and engineering. They often occur in, for example, fluid dynamics, quantum mechanics, chemical reactions, electrical networks, etc. A well-known fact is that the solution of such problems has a multiscale character, i.e. there are thin transition layers where the solution varies very rapidly, while away from the layers the solution behaves regularly and varies slowly. For a detailed discussion on the analytical and numerical treatment of SPBVPs we may refer the reader to the books of O'Malley [1], Doolan et al. [2], Roos et al. [3], Miller et al. [4] and references therein [5-25]. In this paper, a numerical solution of singularly perturbed BVPs (SPBVPs) using an optimal fitted one-step integration scheme via initial value method is present. The original second order SPBVP is replaced by an asymptotically approximate first order initial value singularly perturbed problem (IVSPP) and solved by using an optimal fitted one-step integration scheme. The error analysis is present. Some SPBVPs are solved and the numerical results confirm that the suggested method is accurate and efficient in solving the considered SPBVPs.

\section{DESCRIPTION OF THE METHOD}

Consider the two point second order SPBVP

$$
\varepsilon y^{\prime \prime}+p(x) y^{\prime}+q(x, y)=h(x), \quad x \in[0,1],
$$

$$
y(0)=\alpha \text { and } y(1)=\beta,
$$

Where $0<\varepsilon \ll 1, \alpha$ and $\beta$ are given constants, $p(x)$, $q(x, y)$ and $h(x)$ are assumed to be sufficiently continuously differentiable functions, and $p(x) \geq M>0$ for $x \in[0,1]$ where $M$ is some positive constant. Under these assumptions, the problem (1-2) has a solution which, in general, displays a boundary layer of width $O(\varepsilon)$ at $x=0$.

Following the same procedure suggested by El-Zahar and ELKabeir [9], equation (1) can be written as

$$
\varepsilon y^{\prime \prime}+(p(x) y)^{\prime}=F(x, y),
$$

where $F(x, y)=h(x)+p^{\prime}(x) y-q(x, y)$.

Now, let $u(x)$ be the solution of the reduced problem

$$
p(x) u^{\prime}+q(x, u)=h(x),
$$

with initial condition $u(1)=\beta$.

Also, Eq. (4) can be written as

$$
(p(x) u)^{\prime}=F(x, u),
$$

where $F(x, u)=h(x)+p^{\prime}(x) u-q(x, u)$.

Subtracting Eq.(5) from Eq.(3) and integrating the resulting equation, we have

$$
\int_{1}^{x}\left(\varepsilon \frac{d^{2} y(s)}{d s^{2}}+\frac{d}{d x}(p(s) y(s))\right) d s=\int_{1}^{x}\left(\frac{d}{d x}(p(s) u(s))\right) d s+E,
$$

where

$$
E=\int_{1}^{x}(F(s, y(s))-F(s, u(s))) d s
$$

From (6) and (7), we get

$$
\varepsilon y^{\prime}+p(x) y=p(x) u+K+E, \quad y(0)=\alpha,
$$

where

$$
K=\left(\varepsilon y^{\prime}(s)+p(s)(y(s)-u(s))\right)_{s=1}=\varepsilon y^{\prime}(1)
$$

with boundary conditions 
Lemma 1. Let $y(x)$ and $u(x)$ be respectively the solutions of the SPBVP (1-2) and the reduced problem (4), then

$$
|y(x)-u(x)| \leq C\left(\varepsilon+e^{-M x / \varepsilon}\right), x \in[0,1] .
$$

Proof. See (J. Lorenz [13], theorem 3).

Lemma 2. Let $y(x)$ be the solution of the SPBVP (1-2), then

$$
\left|y^{(m)}(x)\right| \leq C\left(1+\varepsilon^{-m} e^{-M x / \varepsilon}\right), x \in[0,1], m=0,1,2, .
$$

Proof. See (R. Vulanovic [14], theorem1).

With the help of these lemmas 1 and 2 we can prove the following theorem.

Theorem 1. Let $y(x), u(x)$ and $w(x)$ be respectively the solutions of the SPBVP (1-2), the reduced problem (4) and the following initial-value problem

$$
\varepsilon w^{\prime}+p(x) w=p(x) u,
$$

with initial condition $w(0)=\alpha$.

Then,

$$
|y(x)-w(x)| \leq C \varepsilon, \quad \forall x \in[0,1] .
$$

Proof. From Eq.(7) and by using lemma 1 we get the following bounded error

$$
\begin{aligned}
|E| & =\left|\int_{1}^{x}(F(s, y(s))-F(s, u(s))) d s\right|, \\
& \leq \int_{1}^{x}\left(\left|F_{y}(s, \xi)\right||y(s)-u(s)|\right) d s \leq C \varepsilon,
\end{aligned}
$$

where, $\xi$ lies between $u(x)$ and $y(x)$.

Let $m=1$ in lemma 2 , then we get the following bounded error

$$
|K|=\left|\varepsilon y^{\prime}(1)\right| \leq C \varepsilon
$$

Therefore, Eq.(8) becomes

$$
\varepsilon y^{\prime}+p(x) y=p(x) u+O(\varepsilon), \quad y(0)=\alpha,
$$

To estimate the error involved in the solution $w(x)$ of Eq. (9) we proceed as follows

Let $z(x)=y(x)-w(x)$.Then $z(x)$ satisfies the following IVP

$$
\varepsilon z^{\prime}(x)+p(x) z(x)=O(\varepsilon), z(0)=0,
$$

where, $\zeta$ lies between $w(x)$ and $y(x)$.

By integrating Eq. (13), it can be shown that

$$
|z(x)|=|y(x)-w(x)| \leq C \varepsilon .
$$

The proof of theorem 1 is completed.

Thus, by Theorem 1 the solution of the two-point boundary value problem (1-2) is approximated by that of the first order initial value problem (9).

We solve the initial value problem (9) by using an optimal fitted finite difference one-step integration scheme as given in next section.

\section{EXPONENTIALLY FITTED ONE-STEP SCHEME}

Discretizing SPIVP (9) by the Exponentially Fitted One-Step Scheme (EFFD) derived by Salama and Bakr [15] results in the following optimal fitted one-step integration scheme

$$
\frac{\varepsilon}{h}\left(\sigma\left(-\rho \tilde{p}_{j}\right) w_{j+1}-\sigma\left(\rho \tilde{p}_{j}\right) w_{j}\right)=\tilde{p}_{j} u\left(x_{j+1}\right),
$$

where $w_{0}=\alpha, h=1 / N, 0 \leq j<N-1, \rho=h / \varepsilon$,

$\tilde{p}_{j}=\left[p\left(x_{j}\right)+p\left(x_{j+1}\right)\right] / 2, \sigma\left(\rho \tilde{p}_{j}\right)=\rho \tilde{p}_{j} /\left[\exp \left(\rho \tilde{p}_{j}\right)-1\right]$

$\sigma\left(-\rho \tilde{p}_{j}\right)=\rho \tilde{p}_{j} /\left[1-\exp \left(-\rho \tilde{p}_{j}\right)\right]$.

Theorem 2. Let $w(x)$ be the solution of SPIVP (9) and $w_{j}$ be the numerical solution obtained by the two-term recurrence relation (17). Then, at each mesh point $x_{j}$, we have the following error estimate:

$$
\left|w\left(x_{j}\right)-w_{j}\right| \leq C \min \left(h^{2}, \varepsilon\right),
$$

where $C$ is independent of $j, \varepsilon$ and $h$.

Proof. See Salama and Bakr [15], and Doolan et al. [2]

Theorem 3. Let $y(x)$ be the solution of SPBVP (1) and $w_{j}$ be the numerical solution obtained by the two-term recurrence relation (17). Then, at each mesh point $x_{j}$, we have the following error estimate:

$$
\left|y\left(x_{j}\right)-w_{j}\right| \leq C\left(\varepsilon+\min \left(h^{2}, \varepsilon\right)\right),
$$

where $C$ is independent of $j, \varepsilon$ and $h$.

Proof. We have

$$
\left|y\left(x_{j}\right)-w_{j}\right| \leq\left|y\left(x_{j}\right)-w\left(x_{j}\right)\right|+\left|w\left(x_{j}\right)-w_{j}\right|
$$

By applying Theorem 1 and Theorem 2 to the right hand side of the above inequality, we get

$$
\left|y\left(x_{j}\right)-w_{j}\right| \leq C\left(\varepsilon+\min \left(h^{2}, \varepsilon\right)\right) .
$$

The proof of Theorem 3 is completed

\section{NUMERICAL RESULTS}

In this section, five SPBVPs are solved to illustrate the accuracy of the method. These SPBVPs have been discussed in the literature and their approximate solutions are available for comparison. To get more information about the behavior 
International Journal of Engineering Research and Technology. ISSN 0974-3154, Volume 13, Number 5 (2020), pp. 995-1001

(C) International Research Publication House. https://dx.doi.org/10.37624/IJERT/13.5.2020.995-1001

of the solution in the boundary layer region, the solution is computed for $h=2 \varepsilon$ over a narrow region $x_{j} \in[0,20 \varepsilon]$ and $h=0.1$ over the outer region $x_{j} \in(20 \varepsilon, 1]$ at different values of $\varepsilon$

Example 1. Consider the following homogeneous SPBVP $[10,21]$

$$
\varepsilon y^{\prime \prime}(x)+y^{\prime}(x)-y(x)=0, x \in[0,1],
$$

with boundary conditions $y(0)=1$ and $y(1)=1$. The exact solution is given by

$$
y(x)=\frac{\left(e^{m_{2}}-1\right) e^{m_{1} x}+\left(1-e^{m_{1}}\right) e^{m_{2} x}}{e^{m_{2}}-e^{m_{1}}},
$$

where

$m_{1}=(-1+\sqrt{1+4 \varepsilon}) /(2 \varepsilon)$ and $m_{2}=(-1-\sqrt{1+4 \varepsilon}) /(2 \varepsilon)$.
The solution error $\left|y\left(x_{i}\right)-w_{i}\right|_{\infty}$ of Example 1 is shown in Table 1 at different values of the perturbation parameter $\varepsilon$.

Example 2. Consider the following non-homogenous SPBVP from fluid dynamics for fluid of small viscosity [22]

$$
\varepsilon y^{\prime \prime}(x)+y^{\prime}(x)=1+2 x, x \in[0,1],
$$

with boundary conditions $y(0)=0$ and $y(1)=1$. The exact solution is given by

$$
y(x)=x(x+1-2 \varepsilon)+\frac{(2 \varepsilon-1)\left(1-e^{-x / \varepsilon}\right)}{1-e^{-1 / \varepsilon}} .
$$

\begin{tabular}{|c|c|c|c|c|}
\hline Nodes & $\varepsilon=10^{-3}$ & $\varepsilon=10^{-4}$ & $\varepsilon=10^{-5}$ & $\varepsilon=10^{-6}$ \\
\hline $2 \varepsilon$ & $2.4663 \mathrm{e}-4$ & $2.4658 \mathrm{e}-5$ & $2.4657 \mathrm{e}-6$ & $2.4659 \mathrm{e}-7$ \\
\hline $4 \varepsilon$ & $4.2781 \mathrm{e}-4$ & $4.2787 e-5$ & $4.2788 \mathrm{e}-6$ & $4.2790 \mathrm{e}-7$ \\
\hline $6 \varepsilon$ & $4.7247 \mathrm{e}-4$ & $4.7244 \mathrm{e}-5$ & $4.7244 \mathrm{e}-6$ & $4.7246 \mathrm{e}-7$ \\
\hline $8 \varepsilon$ & $4.8140 \mathrm{e}-4$ & $4.8120 \mathrm{e}-5$ & $4.8118 \mathrm{e}-6$ & $4.8120 \mathrm{e}-7$ \\
\hline $10 \varepsilon$ & $4.8317 \mathrm{e}-4$ & $4.8277 \mathrm{e}-5$ & $4.8273 e-6$ & $4.8275 \mathrm{e}-7$ \\
\hline $12 \varepsilon$ & $4.8365 \mathrm{e}-4$ & $4.8306 \mathrm{e}-5$ & $4.8300 \mathrm{e}-6$ & $4.8301 \mathrm{e}-7$ \\
\hline $14 \varepsilon$ & $4.8391 \mathrm{e}-4$ & $4.8312 \mathrm{e}-5$ & $4.8304 \mathrm{e}-6$ & $4.8305 \mathrm{e}-7$ \\
\hline $16 \varepsilon$ & $4.8414 \mathrm{e}-4$ & $4.8315 \mathrm{e}-5$ & $4.8305 \mathrm{e}-6$ & $4.8306 \mathrm{e}-7$ \\
\hline $18 \varepsilon$ & $4.8436 \mathrm{e}-4$ & $4.8318 \mathrm{e}-5$ & $4.8305 \mathrm{e}-6$ & $4.8306 \mathrm{e}-7$ \\
\hline $20 \varepsilon$ & $4.8458 \mathrm{e}-4$ & $4.8320 \mathrm{e}-5$ & $4.8305 e-6$ & $4.8306 \mathrm{e}-7$ \\
\hline 0.1 & $3.6535 \mathrm{e}-4$ & $3.6586 \mathrm{e}-5$ & $3.6591 \mathrm{e}-6$ & $3.6593 \mathrm{e}-7$ \\
\hline 0.2 & $3.5889 \mathrm{e}-4$ & $3.5941 \mathrm{e}-5$ & $3.5946 \mathrm{e}-6$ & $3.5948 \mathrm{e}-7$ \\
\hline 0.3 & $3.4704 \mathrm{e}-4$ & $3.4755 e-5$ & $3.4760 \mathrm{e}-6$ & $3.4763 \mathrm{e}-7$ \\
\hline 0.4 & $3.2873 \mathrm{e}-4$ & $3.2923 \mathrm{e}-5$ & $3.2928 \mathrm{e}-6$ & $3.2931 \mathrm{e}-7$ \\
\hline 0.5 & $3.0274 \mathrm{e}-4$ & $3.0321 \mathrm{e}-5$ & $3.0326 \mathrm{e}-6$ & $3.0328 \mathrm{e}-7$ \\
\hline 0.6 & $2.6765 \mathrm{e}-4$ & $2.6808 \mathrm{e}-5$ & $2.6812 \mathrm{e}-6$ & $2.6814 \mathrm{e}-7$ \\
\hline 0.7 & $2.2184 \mathrm{e}-4$ & $2.2220 \mathrm{e}-5$ & $2.2224 \mathrm{e}-6$ & $2.2226 \mathrm{e}-7$ \\
\hline 0.8 & $1.6344 \mathrm{e}-4$ & $1.6372 \mathrm{e}-5$ & $1.6374 \mathrm{e}-6$ & $1.6376 \mathrm{e}-7$ \\
\hline 0.9 & $9.0308 \mathrm{e}-5$ & $9.0466 \mathrm{e}-6$ & $9.0482 \mathrm{e}-7$ & $9.0489 \mathrm{e}-8$ \\
\hline 1.0 & 0 & 0 & 0 & 0 \\
\hline
\end{tabular}

The solution error of Example 2 is shown in Table 2 at different values of the perturbation parameter $\varepsilon$.

Table 1. Solution error of Example 1 at different values $\varepsilon$ 
International Journal of Engineering Research and Technology. ISSN 0974-3154, Volume 13, Number 5 (2020), pp. 995-1001

(C) International Research Publication House. https://dx.doi.org/10.37624/IJERT/13.5.2020.995-1001

Table 2. Solution error of Example 2 at different values $\varepsilon$

\begin{tabular}{crrrr}
\hline Nodes & $\varepsilon=10^{-3}$ & $\varepsilon=10^{-4}$ & $\varepsilon=10^{-5}$ & \multicolumn{1}{c}{$\varepsilon=10^{-6}$} \\
\hline $2 \varepsilon$ & $1.9965 \mathrm{e}-3$ & $1.9997 \mathrm{e}-4$ & $2.0000 \mathrm{e}-5$ & $2.0000 \mathrm{e}-6$ \\
$4 \varepsilon$ & $2.2644 \mathrm{e}-3$ & $2.2700 \mathrm{e}-4$ & $2.2706 \mathrm{e}-5$ & $2.2707 \mathrm{e}-6$ \\
$6 \varepsilon$ & $2.2982 \mathrm{e}-3$ & $2.3064 \mathrm{e}-4$ & $2.3072 \mathrm{e}-5$ & $2.3073 \mathrm{e}-6$ \\
$8 \varepsilon$ & $2.3004 \mathrm{e}-3$ & $2.3111 \mathrm{e}-4$ & $2.3121 \mathrm{e}-5$ & $2.3122 \mathrm{e}-6$ \\
$10 \varepsilon$ & $2.2984 \mathrm{e}-3$ & $2.3115 \mathrm{e}-4$ & $2.3128 \mathrm{e}-5$ & $2.3129 \mathrm{e}-6$ \\
$12 \varepsilon$ & $2.2957 \mathrm{e}-3$ & $2.3113 \mathrm{e}-4$ & $2.3128 \mathrm{e}-5$ & $2.3130 \mathrm{e}-6$ \\
$14 \varepsilon$ & $2.2930 \mathrm{e}-3$ & $2.3110 \mathrm{e}-4$ & $2.3128 \mathrm{e}-5$ & $2.3130 \mathrm{e}-6$ \\
$16 \varepsilon$ & $2.2902 \mathrm{e}-3$ & $2.3108 \mathrm{e}-4$ & $2.3128 \mathrm{e}-5$ & $2.3130 \mathrm{e}-6$ \\
$18 \varepsilon$ & $2.2875 \mathrm{e}-3$ & $2.3105 \mathrm{e}-4$ & $2.3128 \mathrm{e}-5$ & $2.3130 \mathrm{e}-6$ \\
$20 \varepsilon$ & $2.2847 \mathrm{e}-3$ & $2.3102 \mathrm{e}-4$ & $2.3128 \mathrm{e}-5$ & $2.3130 \mathrm{e}-6$ \\
0.1 & $1.8000 \mathrm{e}-3$ & $1.8000 \mathrm{e}-4$ & $1.8000 \mathrm{e}-5$ & $1.8000 \mathrm{e}-6$ \\
0.2 & $1.6000 \mathrm{e}-3$ & $1.6000 \mathrm{e}-4$ & $1.6000 \mathrm{e}-5$ & $1.6000 \mathrm{e}-6$ \\
0.3 & $1.4000 \mathrm{e}-3$ & $1.4000 \mathrm{e}-4$ & $1.4000 \mathrm{e}-5$ & $1.4000 \mathrm{e}-6$ \\
0.4 & $1.2000 \mathrm{e}-3$ & $1.2000 \mathrm{e}-4$ & $1.2000 \mathrm{e}-5$ & $1.2000 \mathrm{e}-6$ \\
0.5 & $1.0000 \mathrm{e}-3$ & $1.0000 \mathrm{e}-4$ & $1.0000 \mathrm{e}-5$ & $1.0000 \mathrm{e}-6$ \\
0.6 & $8.0000 \mathrm{e}-4$ & $8.0000 \mathrm{e}-5$ & $8.0000 \mathrm{e}-6$ & $8.0000 \mathrm{e}-7$ \\
0.7 & $6.0000 \mathrm{e}-4$ & $6.0000 \mathrm{e}-5$ & $6.0000 \mathrm{e}-6$ & $6.0000 \mathrm{e}-7$ \\
0.8 & $4.0000 \mathrm{e}-4$ & $4.0000 \mathrm{e}-5$ & $4.0000 \mathrm{e}-6$ & $4.0000 \mathrm{e}-7$ \\
0.9 & $2.0000 \mathrm{e}-4$ & $2.0000 \mathrm{e}-5$ & $2.0000 \mathrm{e}-6$ & $2.0000 \mathrm{e}-7$ \\
1.0 & 0 & 0 & 0 & $1.1102 \mathrm{e}-16$ \\
\hline
\end{tabular}

Table 3. Solution error of Example 3 at different values $\varepsilon$

\begin{tabular}{ccccc}
\hline Nodes & $\varepsilon=10^{-3}$ & $\varepsilon=10^{-4}$ & $\varepsilon=10^{-5}$ & $\varepsilon=10^{-6}$ \\
\hline $2 \varepsilon$ & $6.7803 \mathrm{e}-5$ & $6.7681 \mathrm{e}-6$ & $6.7669 \mathrm{e}-7$ & $6.7668 \mathrm{e}-8$ \\
$4 \varepsilon$ & $7.7279 \mathrm{e}-5$ & $7.6871 \mathrm{e}-6$ & $7.6830 \mathrm{e}-7$ & $7.6826 \mathrm{e}-8$ \\
$6 \varepsilon$ & $7.8859 \mathrm{e}-5$ & $7.8144 \mathrm{e}-6$ & $7.8073 \mathrm{e}-7$ & $7.8066 \mathrm{e}-8$ \\
$8 \varepsilon$ & $7.9370 \mathrm{e}-5$ & $7.8345 \mathrm{e}-6$ & $7.8244 \mathrm{e}-7$ & $7.8234 \mathrm{e}-8$ \\
$10 \varepsilon$ & $7.9737 \mathrm{e}-5$ & $7.8402 \mathrm{e}-6$ & $7.8270 \mathrm{e}-7$ & $7.8257 \mathrm{e}-8$ \\
$12 \varepsilon$ & $8.0085 \mathrm{e}-5$ & $7.8439 \mathrm{e}-6$ & $7.8276 \mathrm{e}-7$ & $7.8260 \mathrm{e}-8$ \\
$14 \varepsilon$ & $8.0433 \mathrm{e}-5$ & $7.8473 \mathrm{e}-6$ & $7.8280 \mathrm{e}-7$ & $7.8261 \mathrm{e}-8$ \\
$16 \varepsilon$ & $8.0782 \mathrm{e}-5$ & $7.8507 \mathrm{e}-6$ & $7.8284 \mathrm{e}-7$ & $7.8261 \mathrm{e}-8$ \\
$18 \varepsilon$ & $8.1133 \mathrm{e}-5$ & $7.8541 \mathrm{e}-6$ & $7.8287 \mathrm{e}-7$ & $7.8262 \mathrm{e}-8$ \\
$20 \varepsilon$ & $8.1486 \mathrm{e}-5$ & $7.8575 \mathrm{e}-6$ & $7.8290 \mathrm{e}-7$ & $7.8262 \mathrm{e}-8$ \\
0.1 & 0 & 0 & 0 & 0 \\
0.2 & 0 & 0 & 0 & 0 \\
0.3 & 0 & 0 & 0 & 0 \\
0.4 & 0 & 0 & 0 & 0 \\
0.5 & 0 & 0 & 0 & 0 \\
0.6 & 0 & 0 & 0 & 0 \\
0.7 & 0 & 0 & 0 & 0 \\
0.8 & 0 & 0 & 0 & 0 \\
0.9 & 0 & 0 & 0 & 0 \\
1.0 & 0 & 0 & 0 \\
\hline
\end{tabular}


International Journal of Engineering Research and Technology. ISSN 0974-3154, Volume 13, Number 5 (2020), pp. 995-1001

(C) International Research Publication House. https://dx.doi.org/10.37624/IJERT/13.5.2020.995-1001

Example3. Consider the variable coefficient SPBVP [23,24]

$$
\varepsilon y^{\prime \prime}(x)+\left(1-\frac{x}{2}\right) y^{\prime}(x)-\frac{1}{2} y(x)=0 ; \quad x \in[0,1],
$$

with boundary conditions $y(0)=0$ and $y(1)=1$. The exact solution is approximated in [24] as

$$
y(x)=\frac{1}{2-x}-\frac{1}{2} e^{-\left(x-x^{2} / 4\right) / \varepsilon}
$$

By considering the given problem solution as our exact solution, Tables 3 presents the maximum absolute error $\left|y\left(x_{i}\right)-w_{i}\right|_{\infty}$ for the numerical solution of Example 3 at different values of $\varepsilon$.
Example 4.Consider the non-linear SPBVP [21]

$$
\varepsilon y^{\prime \prime}(x)+2 y^{\prime}(x)+e^{y(x)}=0, \quad x \in[0,1]
$$

with boundary conditions $y(0)=0$ and $y(1)=1$. The problem (23) has a uniformly valid approximation [21] for comparison,

$$
y(x)=\log _{e}(2 /(1+x))-\left(\log _{e} 2\right) e^{-2 x / \varepsilon} .
$$

\begin{tabular}{|c|c|c|c|c|}
\hline Nodes & $\varepsilon=10^{-3}$ & $\varepsilon=10^{-4}$ & $\varepsilon=10^{-5}$ & $\varepsilon=10^{-6}$ \\
\hline $2 \varepsilon$ & $3.6595 \mathrm{e}-5$ & $3.6628 \mathrm{e}-6$ & $3.6631 \mathrm{e}-7$ & $3.6631 \mathrm{e}-8$ \\
\hline $4 \varepsilon$ & $3.7192 \mathrm{e}-5$ & $3.7291 \mathrm{e}-6$ & $3.7301 \mathrm{e}-7$ & $3.7302 \mathrm{e}-8$ \\
\hline $6 \varepsilon$ & $3.7130 \mathrm{e}-5$ & $3.7296 \mathrm{e}-6$ & $3.7313 \mathrm{e}-7$ & $3.7314 \mathrm{e}-8$ \\
\hline $8 \varepsilon$ & $3.7057 \mathrm{e}-5$ & $3.7289 \mathrm{e}-6$ & $3.7312 \mathrm{e}-7$ & $3.7314 \mathrm{e}-8$ \\
\hline $10 \varepsilon$ & $3.6983 \mathrm{e}-5$ & $3.7281 \mathrm{e}-6$ & $3.7311 \mathrm{e}-7$ & $3.7314 \mathrm{e}-8$ \\
\hline $12 \varepsilon$ & $3.6910 \mathrm{e}-5$ & $3.7274 \mathrm{e}-6$ & $3.7311 \mathrm{e}-7$ & $3.7314 \mathrm{e}-8$ \\
\hline $14 \varepsilon$ & $3.6837 \mathrm{e}-5$ & $3.7266 \mathrm{e}-6$ & $3.7310 \mathrm{e}-7$ & $3.7314 \mathrm{e}-8$ \\
\hline $16 \varepsilon$ & $3.6765 \mathrm{e}-5$ & $3.7259 \mathrm{e}-6$ & $3.7309 \mathrm{e}-7$ & $3.7314 \mathrm{e}-8$ \\
\hline $18 \varepsilon$ & $3.6692 \mathrm{e}-5$ & $3.7252 \mathrm{e}-6$ & $3.7308 \mathrm{e}-7$ & $3.7314 \mathrm{e}-8$ \\
\hline $20 \varepsilon$ & $3.6620 \mathrm{e}-5$ & $3.7244 \mathrm{e}-6$ & $3.7308 \mathrm{e}-7$ & $3.7314 \mathrm{e}-8$ \\
\hline 0.1 & 0 & 0 & 0 & 0 \\
\hline 0.2 & 0 & 0 & 0 & 0 \\
\hline 0.3 & 0 & 0 & 0 & 0 \\
\hline 0.4 & 0 & 0 & 0 & 0 \\
\hline 0.5 & 0 & 0 & 0 & 0 \\
\hline 0.6 & 0 & 0 & 0 & 0 \\
\hline 0.7 & 0 & 0 & 0 & 0 \\
\hline 0.8 & 0 & 0 & 0 & 0 \\
\hline 0.9 & 0 & 0 & 0 & 0 \\
\hline 1.0 & 0 & 0 & 0 & 0 \\
\hline
\end{tabular}

The solution error of Example 4 is shown in Table 4 at different values of the perturbation parameter $\varepsilon$.

Table 4. Solution error of Example 4 at different values $\varepsilon$ 
International Journal of Engineering Research and Technology. ISSN 0974-3154, Volume 13, Number 5 (2020), pp. 995-1001

(C) International Research Publication House. https://dx.doi.org/10.37624/IJERT/13.5.2020.995-1001

Table 5. Solution error of Example 5 at different values $\varepsilon$

\begin{tabular}{|c|c|c|c|c|}
\hline Nodes & $\varepsilon=10^{-3}$ & $\varepsilon=10^{-4}$ & $\varepsilon=10^{-5}$ & $\varepsilon=10^{-6}$ \\
\hline $2 \varepsilon$ & $2.6959 \mathrm{e}-4$ & $2.7056 e-5$ & $2.7066 \mathrm{e}-6$ & $2.7067 \mathrm{e}-7$ \\
\hline $4 \varepsilon$ & $1.5996 \mathrm{e}-4$ & $1.6069 \mathrm{e}-5$ & $1.6077 \mathrm{e}-6$ & $1.6078 \mathrm{e}-7$ \\
\hline $6 \varepsilon$ & $2.7986 \mathrm{e}-4$ & $2.8225 \mathrm{e}-5$ & $2.8249 \mathrm{e}-6$ & $2.8251 \mathrm{e}-7$ \\
\hline $8 \varepsilon$ & $3.0348 \mathrm{e}-4$ & $3.0715 \mathrm{e}-5$ & $3.0752 \mathrm{e}-6$ & $3.0756 \mathrm{e}-7$ \\
\hline $10 \varepsilon$ & $3.0676 \mathrm{e}-4$ & $3.1157 \mathrm{e}-5$ & $3.1206 \mathrm{e}-6$ & $3.1211 \mathrm{e}-7$ \\
\hline $12 \varepsilon$ & $3.0630 \mathrm{e}-4$ & $3.1222 \mathrm{e}-5$ & $3.1282 \mathrm{e}-6$ & $3.1288 \mathrm{e}-7$ \\
\hline $14 \varepsilon$ & $3.0522 \mathrm{e}-4$ & $3.1222 \mathrm{e}-5$ & $3.1293 \mathrm{e}-6$ & $3.1300 \mathrm{e}-7$ \\
\hline $16 \varepsilon$ & $3.0404 \mathrm{e}-4$ & $3.1211 \mathrm{e}-5$ & $3.1294 \mathrm{e}-6$ & $3.1302 \mathrm{e}-7$ \\
\hline $18 \varepsilon$ & $3.0284 \mathrm{e}-4$ & $3.1199 \mathrm{e}-5$ & $3.1293 \mathrm{e}-6$ & $3.1302 \mathrm{e}-7$ \\
\hline $20 \varepsilon$ & $3.0166 \mathrm{e}-4$ & $3.1187 \mathrm{e}-5$ & $3.1292 \mathrm{e}-6$ & $3.1302 \mathrm{e}-7$ \\
\hline 0.1 & 0 & 0 & 0 & 0 \\
\hline 0.2 & 0 & 0 & 0 & 0 \\
\hline 0.3 & 0 & 0 & 0 & 0 \\
\hline 0.4 & 0 & 0 & 0 & 0 \\
\hline 0.5 & 0 & 0 & 0 & 0 \\
\hline 0.6 & 0 & 0 & 0 & 0 \\
\hline 0.7 & 0 & 0 & 0 & 0 \\
\hline 0.8 & 0 & 0 & 0 & 0 \\
\hline 0.9 & 0 & 0 & 0 & 0 \\
\hline 1.0 & 0 & 0 & 0 & 0 \\
\hline
\end{tabular}

Example 5.Consider the non-linear SPBVP [25] given by

$$
\varepsilon y^{\prime \prime}(x)+y^{\prime}(x)+(y(x))^{2}=0 \quad x \in[0,1]
$$

with boundary conditions $y(0)=0$ and $y(1)=0.5$. The problem (24) has a uniformly valid approximation [25] for comparison,

$$
y(x)=\frac{1}{1+x}-\frac{e^{-x / \varepsilon}}{(1+x)^{2}} .
$$

The solution error of Example 5 is shown in Table 5 at different values of the perturbation parameter $\varepsilon$. The numerical results show that the proposed method approximates the exact solution very well. Moreover, the numerical solution improves in accuracy as the perturbation parameter $\varepsilon$ tends to zero.

\section{CONCLUSIONS}

In this paper, a numerical solution of SPBVPs having left end boundary layer is presented using an optimal fitted one-step integration scheme via initial value method. The original second order SPBVP is replaced by an asymptotically approximate first order IVSPP and solved by an optimal fitted one-step integration scheme. The error analysis is present. Several SPBVPs are solved and the numerical results show that the proposed method approximates the exact solution very well. Moreover, the numerical solution improves in accuracy as the perturbation parameter $\varepsilon$ tends to zero.

\section{ACKNOWLEDGEMENT}

The author would like to thank Prince Sattam bin Abdulaziz University, Deanship of Scientific Research at Prince Sattam bin Abdulaziz University for their continuous support and encouragement.

\section{REFERENCES}

[1] O'Malley, RE. Singular Perturbation Methods/or Ordinary Differential Equations, Springer-Verlag, New York, 1991.

[2] Doolan, EP. Miller, JJH. Schilders, WHA. Uniform Numerical Methods for Problems with Initial and Boundary Layers, Boole Press, Dublin, 1980. 
[3] Roos, H.-G. Stynes, M., Tobiska, L. Numerical Methods for Singularly Perturbed Differential Equations, Springer, Berlin, 1996.

[4] Miller, JJH. O’Riordan, E., Shishkin, G. I. Fitted Numerical Methods for Singular Perturbation Problems, World Scientific, Singapore, 1996.

[5] Habib HM, El-Zahar ER. An algorithm for solving singular perturbation problems with mechanization. Appl Math Comput, 2007, 188:286-302.

[6] El-Zahar, ER. Piecewise approximate analytical solutions of high order singular perturbation problems with a discontinuous source term, International Journal of Differential Equations, vol. 2016, Article ID 1015634, 12 pages, 2016. doi:10.1155/2016/1015634

[7] El-Zahar, ER. Applications of Adaptive Multi step Differential Transform Method to Singular Perturbation Problems Arising in Science and Engineering, Applied Mathematics and Information Sciences, 2015, 9(1), 223-232.

[8] El-Zahar, ER. Approximate analytical solutions of singularly perturbed fourth order boundary value problems using differential transform method, Journal of King Saud University (Science), 2013, 25(3), 257265.

[9] El-Zahar, ER, EL-Kabeir, SM. A new method for solving singularly perturbed boundary value problems, Applied Mathematics and Information Sciences., 2013, 7(3), 927-938

[10] El-Zahar, ER. Approximate analytical solution of singularly perturbed boundary value problems in MAPLE. AIMS Mathematics, 2020, 5(3): 2272-2284. doi: 10.3934/math.2020150

[11] El-Zahar, ER. Machado, JT., Ebaid, A. A New Generalized Taylor-Like Explicit Method for Stiff Ordinary Differential Equations, Mathematics 2019, 7(12),1154;https://doi.org/10.3390/ math7121154

[12] Chamkha, AJ. Rashad, AM. El-Zahar, ER. EL-Mky, HA. Analytical and Numerical Investigation of Fe3O4-Water Nanofluid Flow over a Moveable Plane in a Parallel Stream with High Suction. Energies,2019, 12(1), 1-18

[13] Lorenz,J. Stability and monotonicity properties of stiff quasilinear boundary problems, Univ. Novom Sadu Zb. Rad. Prir.-Mat. Fak. Sec. Mat, 12(1982), 151-175.

[14] Vulanovic,R. A uniform numerical method for quasilinear singular perturbation problems without turning points, Computing, 41(1989), 97-106.

[15] Salama, A. A., and Bakr, S. A. Optimal extended onestep schemes of exponential type for stiff initial-value problems. International Journal of Computer Mathematics, 2004, 81(11), 1363-1379.

[16] H.J. Reinhardt, Singular perturbations of difference methods for linear ordinary differential equations, Appl. Anal. 10 (1980).

[17] Varner, T. N., and Choudhury, S. R. (1998). Nonstandard difference schemes for singular perturbation problems revisited. Applied Mathematics and Computation, 92(2-3), 101-123.

[18] V. Shanthi and N. Ramanujam, A Boundary Value Technique for Boundary Value Problems for Singularly Perturbed Fourth-Order Ordinary Differential Equation, Comp. Math. Appl, 47(2004), 1673-1688.

[19] B.S. Attili, Numerical treatment of singularly perturbed two point boundary value problems exhibiting boundary layers, Commun. Nonlinear Sci. Numer. Simul., 16 (9) (2011), 3504-3511.

[20] Kumar, M. A recent development of computer methods for solving singularly perturbed boundary value problems, International Journal of Differential Equations, 2011 (2011), 1-32.

[21] Bender, C. M. and Orszag, S. A. Advanced mathematical methods for scientists and engineers I, Springer Science \& Business Media, 1999.

[22] Reinhardt, H.J. Singular perturbations of difference methods for linear ordinary differential equations, Appl. Anal. 10 (1980).

[23] Kevorkian, J. Cole, J.D. Perturbation Methods in Applied Mathematics, Springer, New York,1981.

[24] Nayfeh, A.H. Perturbation Methods, Wiley, New York, 1979.

[25] El-Zahar, E. R. Approximate analytical solutions for singularly perturbed boundary value problems by multi-step differential transform method." Applied Sci 12 (2012): 2026-2034. 